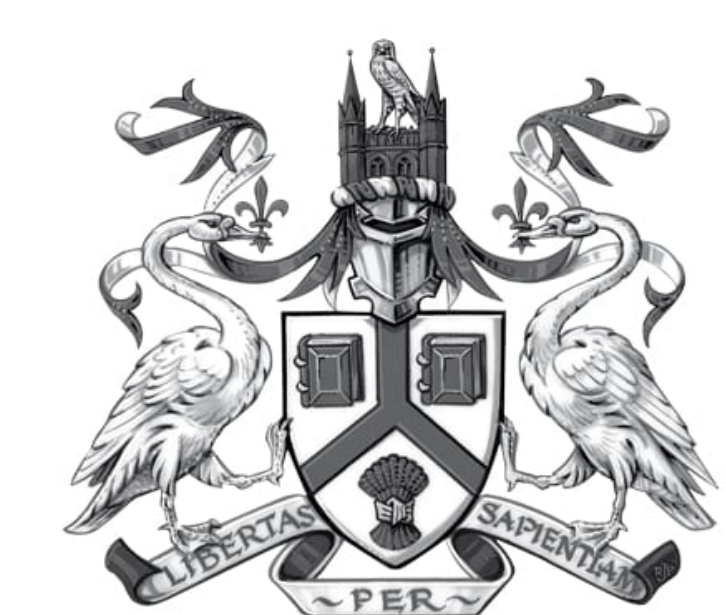

UNIVERSITY OF

\title{
MODEL BASED 3D POINT CLOUD SEGMENTATION FOR AUTOMATED SELECTIVE BROCCOLI
}

HARVESTING

Hector A. Montes, ${ }^{1}$ Grzegorz Cielniak, ${ }^{2}$ and Tom Duckett ${ }^{3}$

'hmontes@lincoln.ac.uk; 2tduckett@lincoln.ac.uk; ${ }^{3}$ GCielniak@lincoln.ac.uk. School of Computer Science, University of Lincoln, Brayford Pool, Lincoln, LN6 7TS, UK.

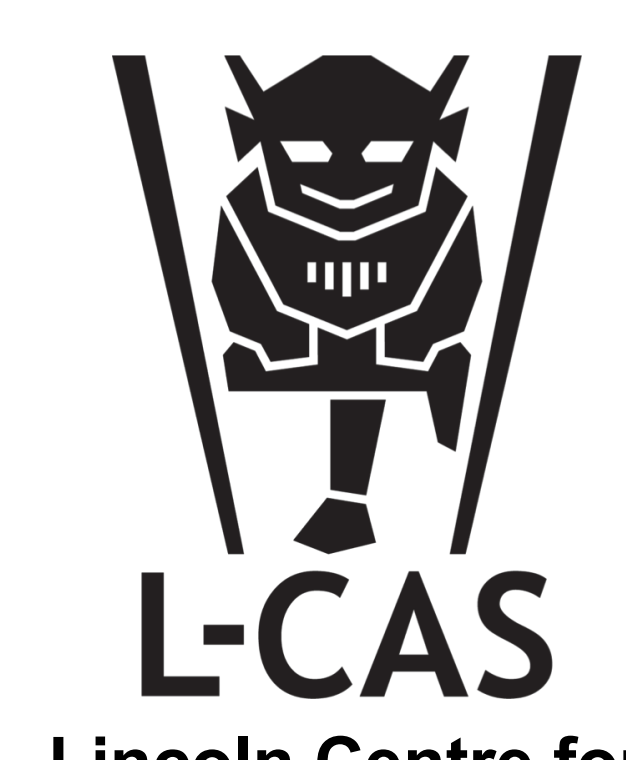
Autonomous Systems

\section{PROBLEM BACKGROUND}

Segmentation of 3D objects in cluttered scenes is a highly relevant problem. Given a 3D point cloud produced by a depth sensor, the goal is to separate objects of interest in the foreground from other elements in the background.

We research 3D imaging methods to accurately segment and identify broccoli plants in the field. The ability to separate parts into different sets of sensor readings is an important task towards this goal. Our research is focused on the broccoli head segmentation problem as a first step towards size estimation of each broccoli crop in order to establish whether or not it is suitable for cutting.

\section{METHODOLOGY}

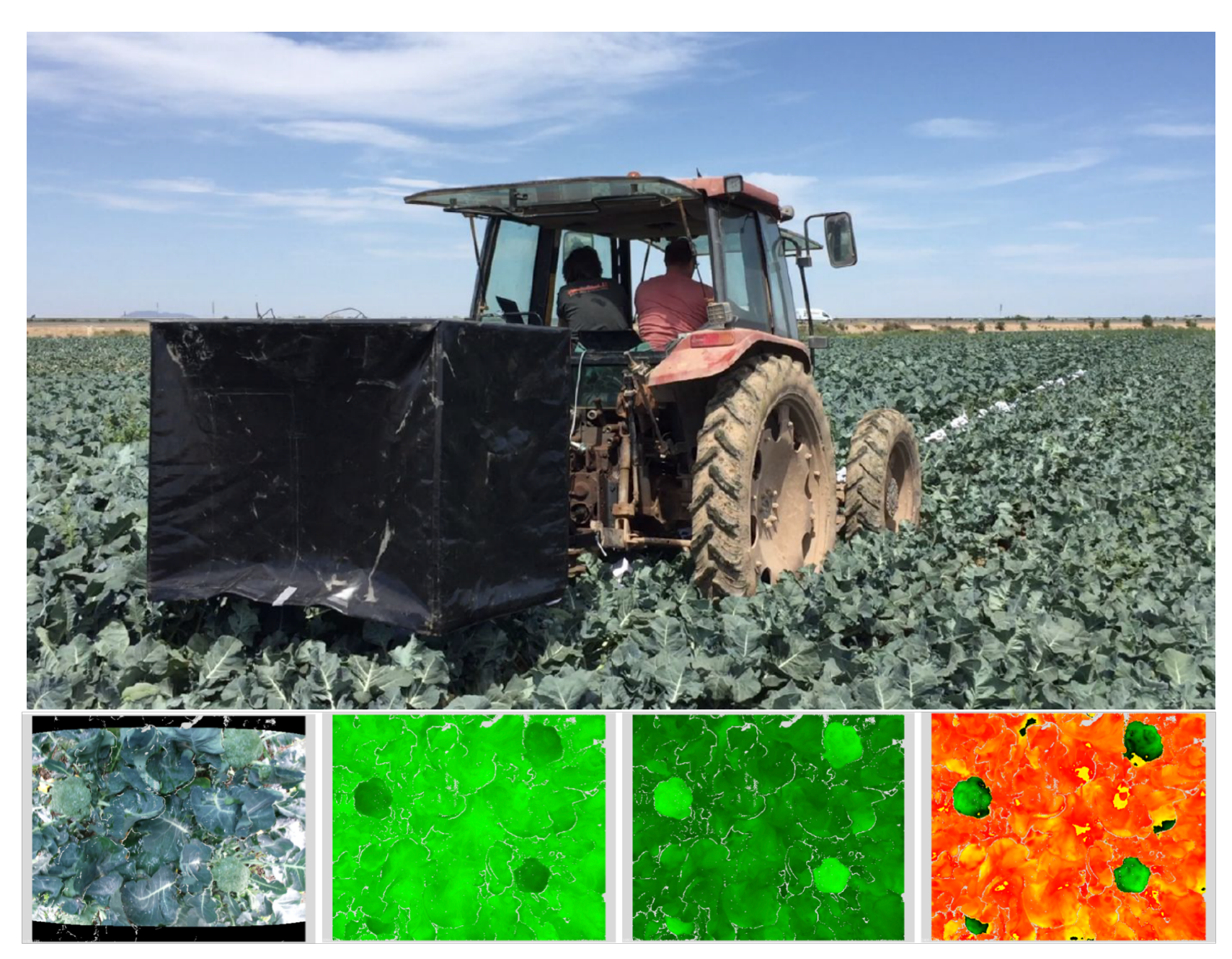

Data acquisition

The 3D point cloud data was captured in outdoor fields under different weather conditions in 4 locations: 2 in the UK, 1 in Spain, and 1 more in USA using the Kinect 2 sensor

Model based 3D point cloud segmentation

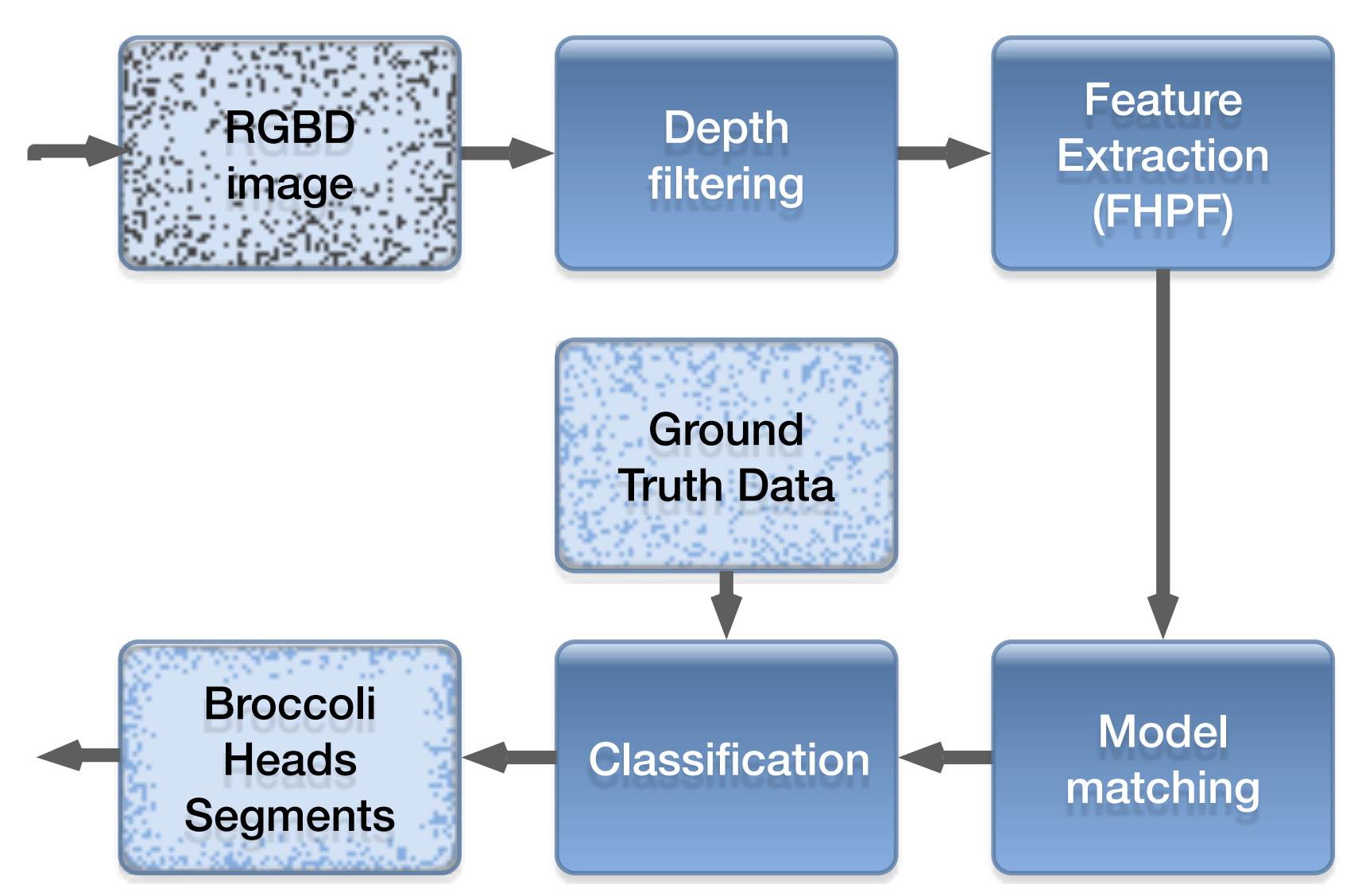

Segmentation system pipeline The frames of 3D point cloud data are first filtered by depth. Then features are extracted from each point and matched to the reference models. The points with the same target class are grouped to form the final segments
Reference models

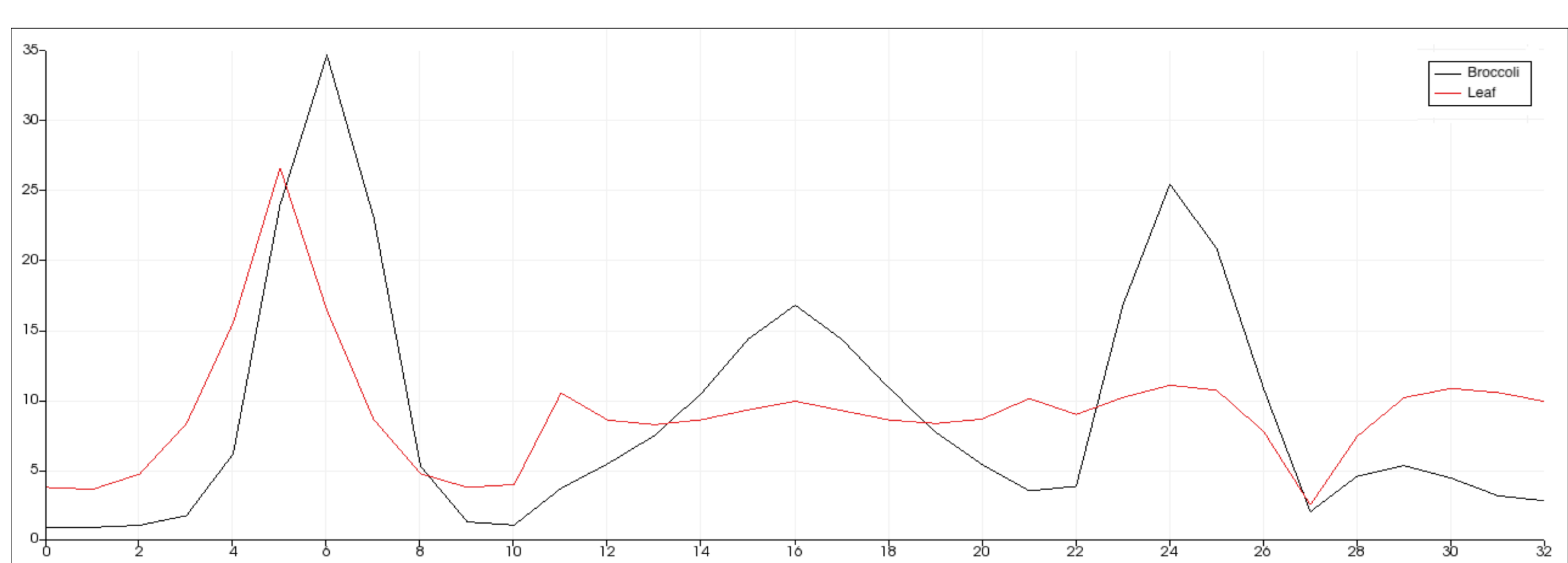

Histograms of the reference models used in our algorithm. A FPFH descriptor [1], based on a set of angular features, is computed for each data point. The descriptor is then matched to both reference models and the difference provides the final classification score.

\section{PRELIMINARY EXPERIMENTAL RESULTS}

To evaluate our results, we compare our experiments to those reported by Kusumam et al. [2]. We focus on frames where those experiments produced either under segmentation, i.e some of the the extracted segments were larger than the broccoli heads seen in the frame.

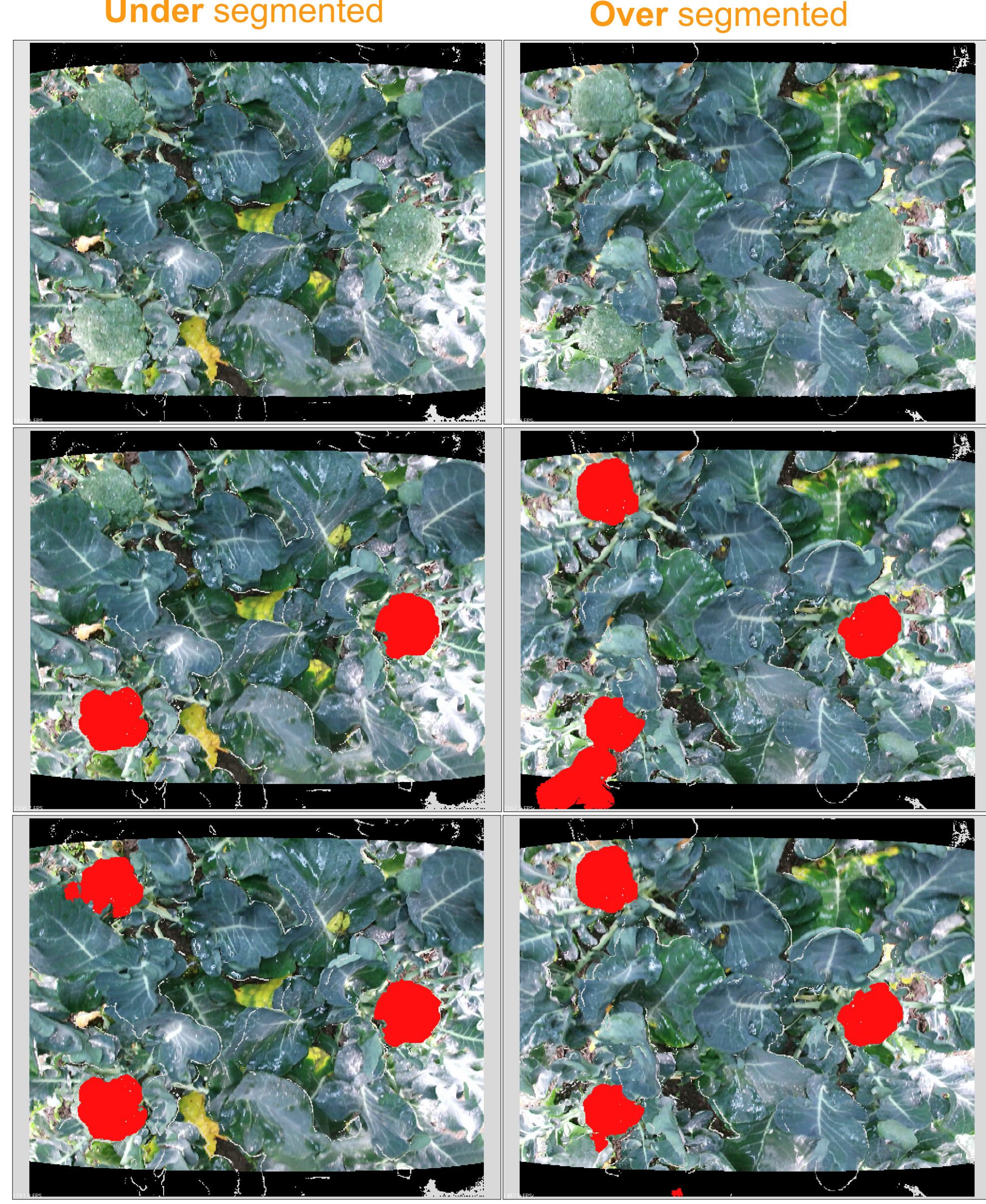

Broccoli segmentation steps

Top row: The original frame on the left, and after been depth filtered on the right. Mid row: matched points of broccoli heads shown in bright green on the left, and other points shown on the right. Bottom row: The difference of the two reference models shown in contrasting colours on the left and, on the right, the extracted segments in red.
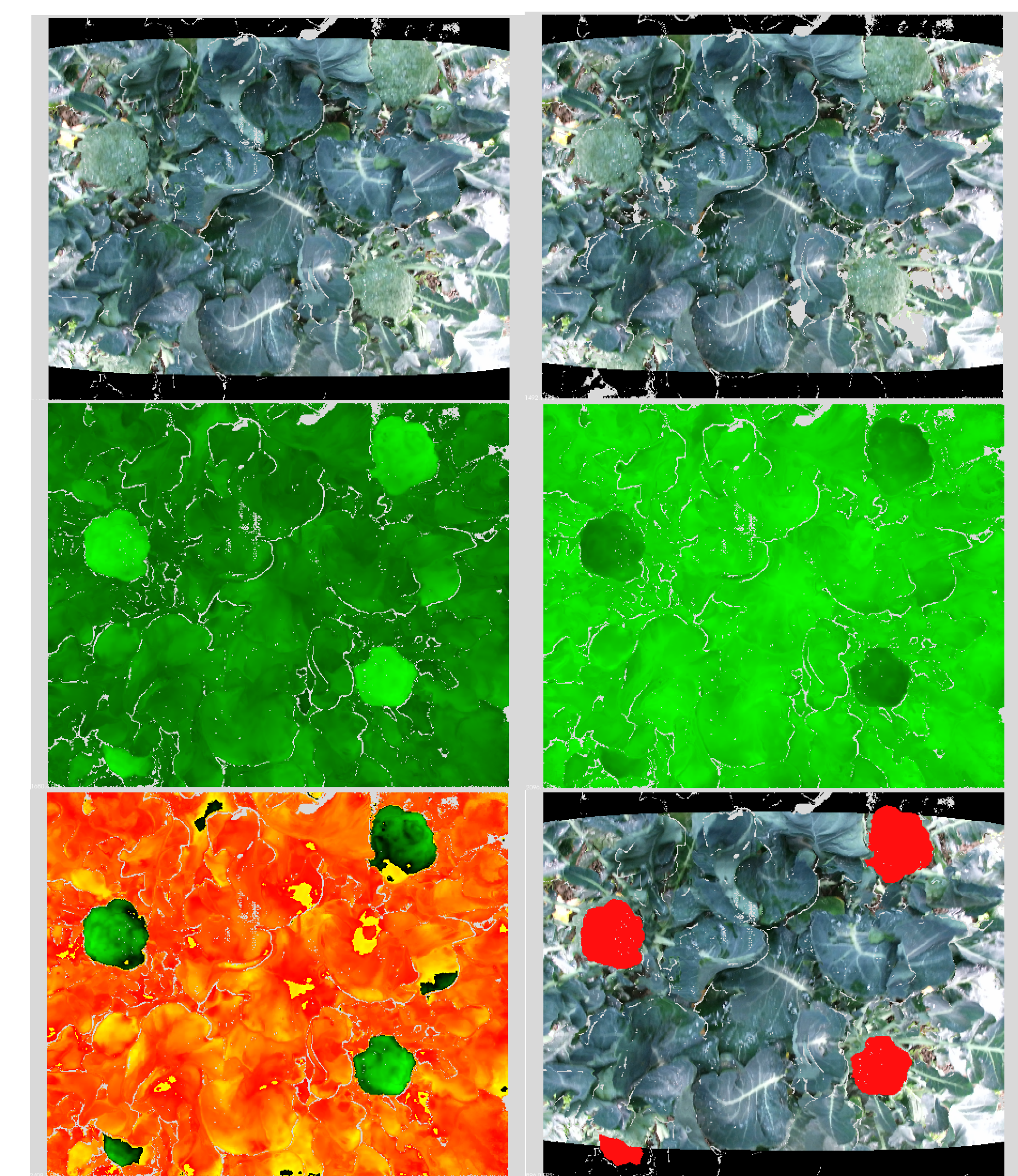

REFERENCES

[1] R. B. Rusu, N. Blodow, and M. Beetz. Fast Point Feature Histograms (FPFH) for 3D Registration. In Proc. IEEE ICRA, Kobe Japan. 2009

[2] K. Kusumam, T. Krajník, S. Pearson, T. Duckett, and G. Cielniak. 3D-vision based detection, localization, and sizing of broccoli heads in the field. Journal of Field Robotics, 34(8):15051518, 2017.

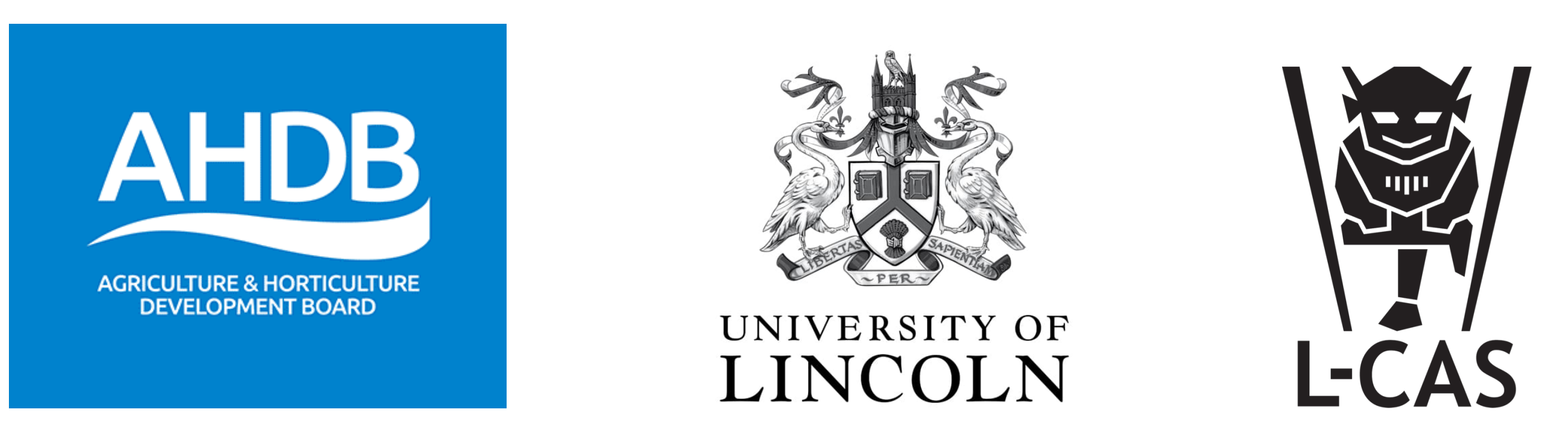

